\title{
Frenilectomía lingual con mioplastia del geniogloso
}

\author{
Lingual frenectomy with myoplasty of the genioglossus
}

\author{
Madeleine Edith Vélez-Cruz,* Raúl Alejandro León-Aguilar, ${ }^{\ddagger}$ Luis Alberto Montoya-Pérez,* \\ Manuel Estrada-Rivera, ${ }^{\S}$ Raúl José Vives-Serrano, " Carlos Juan Liceaga-Escalera* \\ * Servicio de Cirugía Maxilofacial; ${ }^{\ddagger}$ Residente de 3er año en Cirugía Maxilofacial; § Servicio Otorrinolaringología; \\ " Cirujano Maxilofacial. Hospital Juárez de México, Ciudad de México, México.
}

\begin{abstract}
RESUMEN
La anquiloglosia es la condición en la que existe un frenillo sublingual que cambia la apariencia o la función de la lengua. El tratamiento generalmente es quirúrgico.

Se presenta el caso de un niño de cuatro años de edad a quien se le realizó mioplastia del músculo geniogloso, que consiste en realizar un corte trasversal sobre el eje longitudinal del músculo, lo que permite la elongación de la lengua. El resultado fue exitoso, ya que seis meses después del procedimiento hubo mejoría en la amplitud de los movimientos de la lengua y para articular palabras.
\end{abstract}

Palabras clave: Anquiloglosia, mioplastia, frenilectomía, dislalia, preescolar.

\section{INTRODUCCIÓN}

Los frenillos bucales son estructuras tisulares que se presentan como vestigios del desarrollo de la cavidad bucal; son complexiones dinámicas que están sujetas a variaciones en forma, tamaño y posición durante las distintas etapas del crecimiento del individuo. ${ }^{1}$

La Academia Americana de Medicina en Lactancia Materna define a la anquiloglosia como la condición física en la que existe un frenillo sublingual que cambia la apariencia o función de la lengua, debido a su longitud disminuida, falta de elasticidad o fijación demasiado distal en ese músculo, o demasiado cerca de la cresta

\begin{abstract}
Ankyloglossia is a condition in which there is a sublingual frenulum which changes the appearance and function of the tongue. Treatment is usually surgical.

The case reported is of a four-year-old who underwent myoplasty of the genioglossus muscle, which involves making a crosscut on the longitudinal axis of the muscle, allowing the tongue to elongate. The surgical result was successful since, six months after the procedure, an improvement was determined in the range of movements of the tongue and in the articulation of words.
\end{abstract}

Keywords: Ankyloglossia, myoplasty, frenectomy, dyslalia, toddler.

gingival cuando es incompleta, y completa cuando se presenta un anclaje prácticamente total de la lengua al piso de la boca. ${ }^{2}$

En la población general, los frenillos de la lengua tienen una prevalencia de 0.02 a 4.8\%; esta variación se cree que es debido a una falta de uniformidad y estandarización de los métodos diagnósticos. La relación hombre-mujer es diferente, de acuerdo con el reporte consultado, los datos varían de 2.6:1.0 y de $1.5: 1.0 .^{3-5}$

Para fines prácticos, en el presente artículo se utilizó la escala de Kotlow de 1999, que evalúa la "lengua libre", que es la longitud desde la inserción del frenillo

Correspondencia: Raúl Alejandro León-Aguilar, drleonmaxilofacial@outlook.com

Citar como: Vélez-Cruz ME, León-Aguilar RA, Montoya-Pérez LA, Estrada-Rivera M, Vives-Serrano RJ, Liceaga-Escalera CJ. Frenilectomía lingual con mioplastia del geniogloso. Rev Mex Pediatr. 2021; 88(2): 67-70. https://dx.doi.org/10.35366/101280 
lingual en la cara ventral de la lengua por su base, hasta la punta de ese órgano. ${ }^{6}$

El tratamiento es quirúrgico; la mioplastia del músculo geniogloso es una técnica descrita en 1968 por el Dr. Javier Sánchez Torres, que consiste en realizar una incisión trasversal sobre el eje longitudinal del músculo, lo que permite un punto de mayor elongación de la lengua y que, generalmente, se ejecuta en conjunto con otras técnicas buscando la obtención de mejores resultados. ${ }^{7}$ En comparación con otras, esta cirugía tiene como ventaja que es relativamente sencilla, sin un aumento significativo de morbilidad del sitio quirúr- gico. Se presenta el caso de un niño a quien se realizó este procedimiento y que resultó exitoso.

\section{PRESENTACIÓN DEL CASO}

Se trata de un paciente masculino de cuatro años de edad, con el diagnóstico de anquiloglosia grado III de Kotlow, con $5 \mathrm{~mm}$ de longitud lingual y un diagnóstico foniátrico de dislalia (Figura 1A).

Bajo anestesia general balanceada, previa asepsia y antisepsia, se realizó marcaje en el sitio quirúrgico, tomando a modo de referencias el punto más distal y la
Figura 1:

A) Glosorrafia (se utiliza una sonda de alimentación infantil para cubrir la seda y proteger la mucosa del vientre lingual). Se aprecia el frenillo lingual fibromuscular con inserción anómala (Kotlow III). B) Frenotomía romboidal.
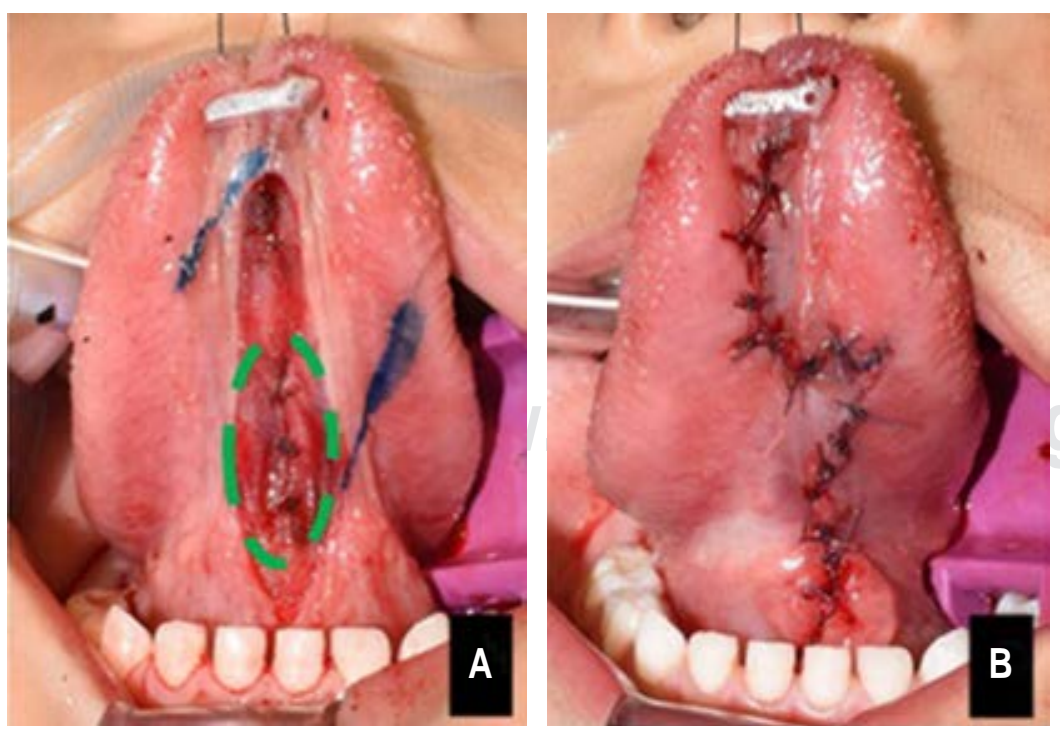

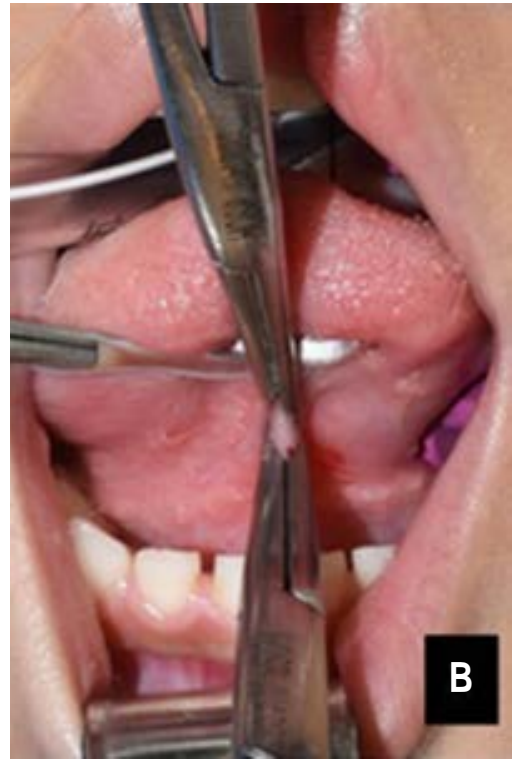

Figura 2:

A) Sutura de las fibras del músculo geniogloso en sentido vertical, las cuales previamente se incidieron en sentido horizontal. B) Zetaplastia del vientre lingual. 

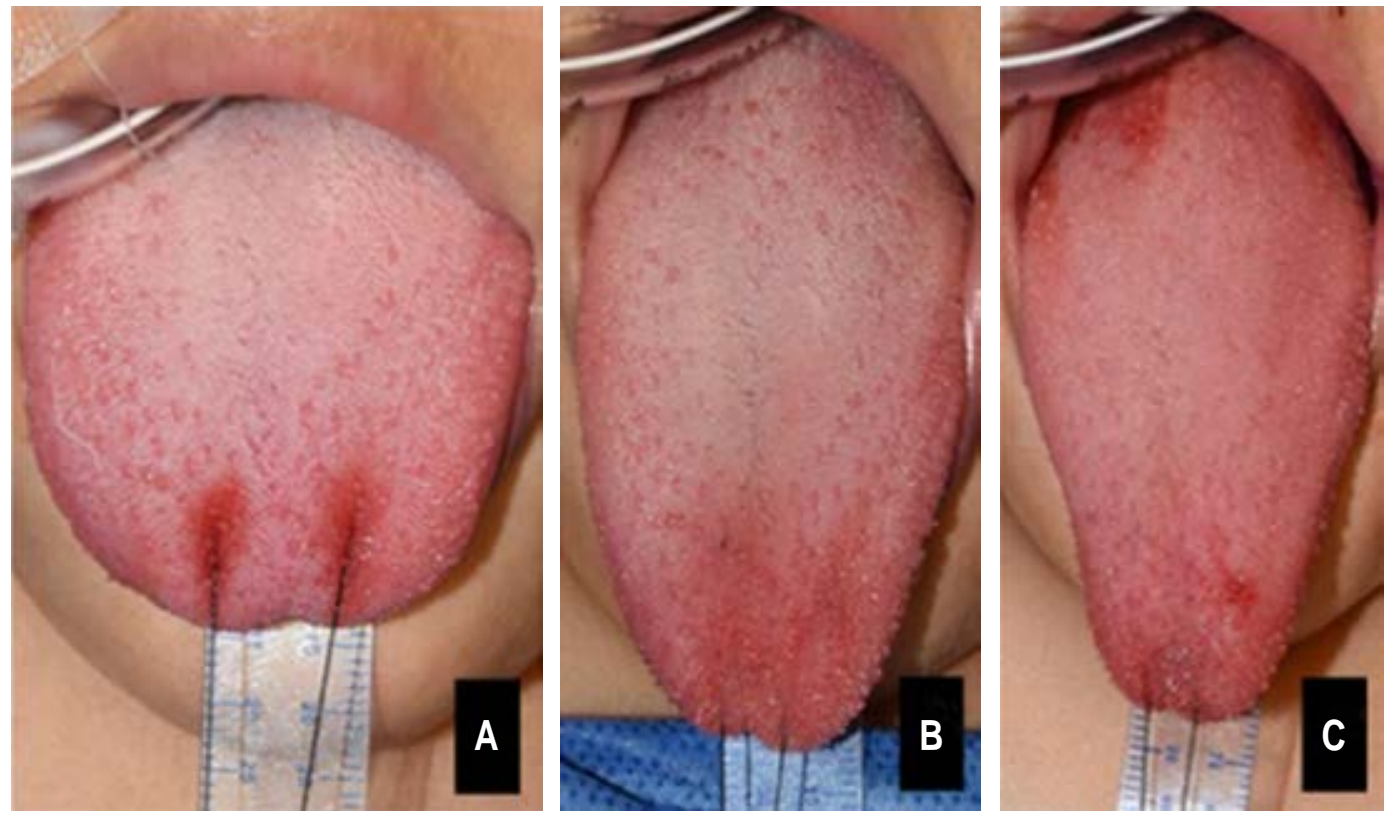

Figura 3:

Medición
transoperatoria de la
elongación lingual,
colocando una regla
milimétrica en el
surco mentolabial,
a fin de que se
pudiera reubicar
en el mismo punto
de manera precisa.
A) Situación inicial.
B) Mioplastia del
geniogloso. Aumento
de aproximadamente
11 mm. C) Zetaplastia
ventral suturada.
Incremento final de
alrededor de 17 mm.
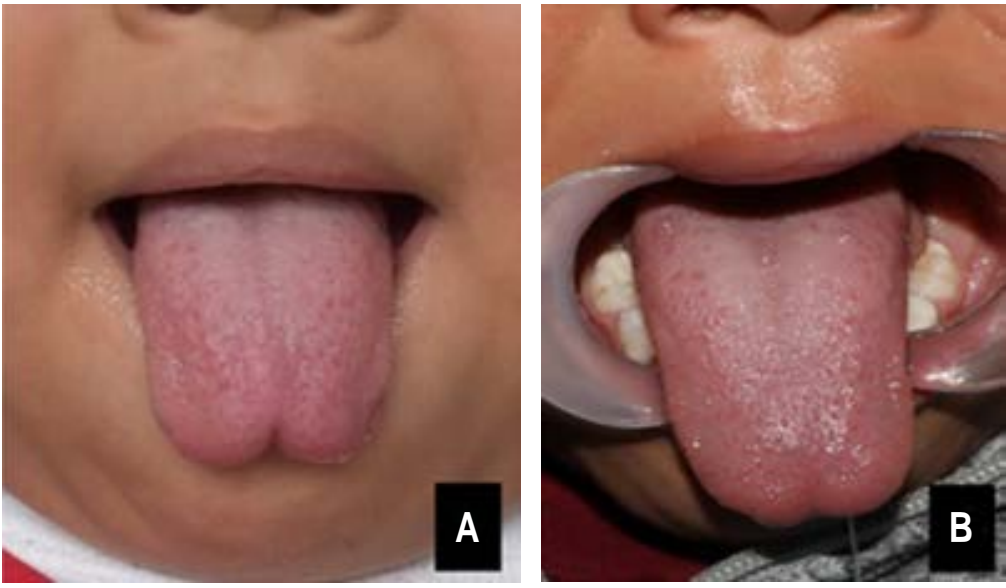

Figura 4:

A) Proyección lingual prequirúrgica. B) Resultado postquirúrgico con una extensión en la proyección lingual de aproximadamente $17 \mathrm{~mm}$.

base del frenillo lingual. Como primer paso, se abordó la frenectomía con técnica romboidal del cuerpo de dicho pliegue cutáneo, con ayuda de dos pinzas hemostáticas posicionadas de manera convergente hacia la base de la lengua, efectuando la escisión quirúrgica del frenillo (Figura 1B).

Posteriormente, se hizo incisión en sentido transversal al eje longitudinal del músculo geniogloso de aproximadamente $1 \mathrm{~cm}$ de longitud, y se suturaron las fibras de manera vertical (Figura 2A). Se disecó la mucosa ventral y se realizaron dos incisiones para z-plastia; la primera iniciando del punto más distal del frenillo con dirección hacia la derecha de aproximadamente $2 \mathrm{~cm}$ de longitud. La segunda incisión de la base del citado frenillo hacia la izquierda de aproximadamente $2 \mathrm{~cm}$ de longitud con una angulación de aproximadamente $45^{\circ}$, se reposicionan y se realizó sinéresis, con sutura de ácido poliglicólico 3-0 (Figura $2 B)$. La medición de la elongación lingual obtenida con cada paso se muestra en la Figura 3.

El paciente durante el periodo postoperatorio cursó con dolor leve con escala EVA (3/10), con notable incremento del rango de movimientos de la lengua e inicio de terapia durante las primeras horas. En el seguimiento a seis meses de la intervención quirúrgica, se observó aumento significativo en la amplitud de movimientos (Figura 4), de $17 \mathrm{~mm}$ al inicio y de $22 \mathrm{~mm}$ al final. Asimismo, con respecto a la dinámica lingual se observó 
mejoría para la articulación de las palabras, con un incremento gradual de la calidad fonética.

\section{DISCUSIÓN}

Con la utilización en conjunto de distintas técnicas para la corrección de alteraciones estructurales del frenillo lingual, observamos que mediante un solo acto operatorio se proporcionan óptimos resultados en la dinámica lingual. Se considera que es una buena alternativa para el tratamiento de anquiloglosia grave cuando los pacientes se encuentran en estadio III y IV de Kotlow.

El método quirúrgico aquí descrito parece ser seguro, ya que no incrementa el tiempo quirúrgico ni las complicaciones. Sin embargo, el principal inconveniente es que se recomienda llevar a cabo el procedimiento bajo anestesia general, debido a que pacientes de corta edad podrían proporcionar poca colaboración, haciendo que el riesgo quirúrgico de este procedimiento aumente.

\section{REFERENCIAS}

1. Izolani Nieto O, Molero VG, Goulart RM. Frenectomia: revisão de literatura. Revista Uningá Review. 2014; 18(3): 21-25.

2. Amir LH, James JP, Donath SM. Reliability of the hazelbaker assessment tool for lingual frenulum function. Int Breastfeed $\mathrm{J}$. 2006; $1(1): 3$.

3. Ballard JL, Auer CE, Khoury JC. Ankyloglossia: assessment, incidence, and effect of frenuloplasty on the breastfeeding dyad. Pediatrics. 2002; 110(5): e63.

4. Correa MSNP, Abanto JA, Correa FNP, Bonini GAVC, Alves FBT. Anquiloglosia y amamantamiento: Revisión y reporte de un caso. Rev Estomatol Herediana. 2008; 18(2): 123-127.

5. Messner AH, Lalakea ML, Aby J, Macmahon J, Bair E. Ankyloglossia: incidence and associated feeding difficulties. Arch Otolaryngol Head Neck Surg. 2000; 126(1): 36-39.

6. Kotlow LA. Ankyloglossia (tongue-tie): a diagnostic and treatment quandary. Quintessence Int. 1999; 30(4): 259-262.

7. Sánchez TJ. Resección del frenillo lingual con mioplastia del geniogloso (técnica personal). Rev ADM. 1968; 25(2): 135-140.

Conflicto de intereses: Los autores declaran que no tienen. 